\title{
EDITORIAL
}

\section{Caring for caregivers/care partners of persons with dementia}

According to the World Health Organization (WHO), approximately 50 million people worldwide have dementia, and this number may triple by 2050 (https://www.who.int/news-room/fact-sheets/ detail/dementia). There are nearly 10 million new cases of diagnosable dementia every year. Dementia has a physical, psychosocial, and economic impact, not only on people with dementia but also on their caregivers, families, and the society at large. The WHO has specified support for dementia caregivers as one of its public health priorities. In the US alone, more than 15.5 million people are informal (i.e. unpaid) caregivers for individuals with Alzheimer's disease and related dementias (Alzheimer's Association, 2017). The Alzheimer's Disease International and Karolinska Institute estimate that the annual global number of informal care hours provided to people with dementia living at home was about 82 billion hours in 2015, equivalent to more than 40 million full-time workers, and this will increase to 65 million full-time workers by 2030 (https://www .alzint.org/u/global-estimates-of-informal-care.pdf). A majority $(60 \%)$ of people with dementia live in lowand middle-income countries, and $96 \%$ of people with dementia in those countries live at home.

Family caregivers of people with dementia are the "invisible second patients" and are also central to the quality of life of their loved ones with dementia (Brodaty and Donkin, 2009). While caregiving has some positive psychological effects such as pride and contentedness, it has many negative effects including high levels of stress, burnout, anxiety, and depression. Specifically, recent comprehensive reviews suggest that significant caregiver burden is prevalent in nearly $50 \%$ of caregivers (Collins and Kishita, 2020). Approximately one-third of the caregivers endorse symptoms of depression and anxiety (Kaddour and Kishita, 2020). This emotional strain of caregiving may be due to reduced use of positive coping and greater use of negative coping strategies compared to their noncaregiving peers (Mausbach et al., 2013). Stress and emotional strain in caregivers may lead to physical comorbidities. For example, chronic stress among dementia caregivers is associated with chronic systemic inflammation (Potier et al., 2018), a pathogenic basis for cardiovascular, metabolic, and neurocognitive disorders. Interventions to reduce caregiver burden and depression are necessary to delay nursing home admission as well as for improving mental and physical well-being. Emotionally supportive communication between spouses in the early stages of dementia can protect caregivers' health, and active behavioral interventions for caregivers can improve well-being via reduction in depressive symptoms and systemic inflammation (Moore et al., 2013; von Känel et al., 2020), but clearly more work is needed in this area. Despite the wealth of literature on caregiving-related issues, research is required to help tailor interventions for caregivers so that a significant positive impact on caregivers' and care receivers' well-being can be achieved.

In recent years, it has been suggested that the term caregiver or carer should be replaced by the term "care partner". This view is based on the notion that nonprofessional care of a person, particularly with long-term chronic disease, is a partnership between the person with the disease and significant people in their lives (Bennett et al., 2017). The care partner concept recognizes the key role that people with the chronic condition play in their own care. Accordingly, it is recommended that care partners be included as important members of the health care team in informing policy, education, and research. However, this concept may become somewhat difficult for persons with latestage Alzheimer's disease and other dementias, who are unable to make major decisions for themselves.

This issue of the International Psychogeriatrics includes seven articles, with respective invited commentaries, on different aspects of caregiving for people with dementia. Notably, these articles come from seven different countries: Australia (Murfield et al.), Denmark (Clemmensen et al.), Ireland (Teahan et al.), Norway (Terum et al.), Spain (Madruga et al.), the UK (Pione et al.), and the USA (Jutkowitz et al.).

Teahan et al. studied 2311 family carers of older people. In about $20 \%$ of those cases, the care recipients had a diagnosis of dementia. The findings demonstrated significant differences in carer burden distribution, with family carers of people with dementia being significantly more likely to report moderate or high carer burden. Therefore, family carers of people with dementia may require tailored social supports to maintain good health and 
well-being. Other variables associated with caregiving stress included female gender, higher level of education, parental rather than spousal relationship, living with the care recipient, greater dependency of the care recipient, and low level of perceived support.

The study by Terum et al. included 107 patients admitted to a nursing home with Alzheimer's disease or dementia with Lewy bodies and their primary carers. Carer distress was an important contributor to early nursing home admission, explaining $19 \%$ of the total variance of time until admission. The most important predictors of the latter were feeling frustrated, having limitations on social life, not being able to get away on vacation, and feeling unable to cope with the situation.

Murfield and colleagues studied an international sample of 171 family carers of adults aged 65 years or older. Compassionate Engagement and Action Scales (CEAS) were used to measure compassion of three different types - self-compassion, compassion toward others, and compassion from others. Confirmatory factor analyses lent support for the CEAS as measures of these three varieties of compassion. Within all three orientations, there was support for the measurement of compassion as an overarching construct composed of two dimensions: engagement and actions.

As mentioned above, a large majority of published papers in the literature have appropriately focused on deleterious effects of dementia caregiving. However, the positive effects of being a caregiver also deserve some attention. In that spirit, Pione et al. reviewed 25 measures of positive constructs of resilience, self-efficacy, and positive aspects of caregiving. Noting the limitations of the various studies, the authors found that several specified scales scored high among the instruments within their respective constructs.

Clemmensen and colleagues applied an iterative process of data collection to evaluate the face and content validity of the Dementia Carer Assessment of Support Needs Tool (DeCANT). The authors suggest that this 25-item carer-reported questionnaire may be used to help identify their support needs when caring for a person with dementia. This would enable development and testing of supportive interventions aimed at improving carers' health and well-being.

Madruga et al. conducted a randomized controlled trial (RCT) in 48 women informal primary caregivers, 25 of whom were randomly allocated to the intervention group, and 23 participants to a control group. The intervention involved two 60minute-long physical exercise sessions per week, supervised by a personal trainer at caregivers' home, over nine months. The intervention had a positive impact on caregivers' subjective burden and risk of depression. Care recipients' level of functional independence and area of residence were significant predictors of the efficacy of the intervention. The relatively small sample size suggests a need for replication and expansion of the findings in future trials.

Tailored nonpharmacologic dyadic interventions which provide family caregivers knowledge and skills to manage dementia-related clinical symptoms (e.g. instructing in communication, task, and environmental strategies) reduce dementia-related behavioral symptoms, caregiver stress, and time spent caregiving. Yet, these interventions have not been generally adopted by systems of care, largely due to a lack of a sustainable payment model for such programs, at least in the US. Jutkowitz et al. conducted an RCT in 250 caregiver-care recipient dyads to determine the willingness-to-pay of family caregivers to learn care strategies for persons with dementia. A Tailored Activity Program (TAP), which provided activities tailored to the care recipients, and instructed caregivers in their use, was compared to attention control. Family caregivers were found to be willing to pay more for an intervention immediately following participation in an in-home nonpharmacologic intervention program similar to the one they were asked to value. The authors correctly point to a need for developing innovative payment models to help implement TAP and other nonpharmacologic home-based carer support programs.

The seven studies summarized above cover varied aspects of dementia caregiving and are an excellent addition to the literature. However, there continues to be a need to understand the mechanisms that make interventions more effective for caregivers. The articles by Teahan et al. and Terum et al. suggest a continued need for scientists to understand how interventions can reduce caregiver burden and distress, not just for the caregiver but also for the care recipient. The articles by Murfield et al. and Pione et al. offer examples of positive constructs such as compassion and resilience that may be targeted to help reduce distress and improve overall well-being. Madruga and colleagues provide evidence that supervised exercise may be a simple but effective way to reduce caregiver burden and improve well-being.

Another need for caregiver research is to understand for whom and under what circumstances interventions become most effective. Tailoring interventions to the specific needs of caregivers is the right step to making them most effective. The manuscript by Clemmenson et al. offers a scale to help assess specific caregiver needs, which in turn can help tailor interventions to meet those needs. Finally, the need to make interventions affordable and available to caregivers is long overdue. Jutkowitz 
and colleagues break the mold of caregiver intervention research by examining caregivers' willingness to pay for nonpharmacologic interventions. Information such as this may be of high value to communitybased organizations by helping them select the most efficacious intervention that could offer the caregivers the best value for their money.

There are several other areas that need additional clinical research relevant to dementia caregivers. One such area pertains to social determinants of health like loneliness and social isolation among dementia caregivers. The recent behavioral pandemics of loneliness and loneliness-associated suicides and opioid use-related mortality have caused a reduction in average lifespan - even before the COVID-19 pandemic hit the world (Jeste et al., 2020). Loneliness is associated with increased risk of neurocognitive, metabolic, cardiovascular, and psychiatric disorders and greater all-cause mortality. Over $40 \%$ of caregivers of persons with Alzheimer's disease and related dementias report at least moderate level of loneliness and social isolation (Victor et al., 2020). In the midst of the COVID-19 pandemic, social distancing requirements have increased loneliness of older adults. Yet, we have only a limited array of effective interventions. Technology-based solutions can provide physically distanced social engagement; however, there are additional concerns about digital literacy among older adults including problems with access to devices, usage opportunities, and skill building. Identifying how caregiving stressors and loneliness impact health behaviors (e.g. physical activity, sleep, and diet) may provide avenues to improve the negative health outcomes associated with caregiver burden. Such mechanistic approaches can help personalize interventions for each individual and optimize overall health and quality of life. There is thus a need for research on developing and testing effective and efficacious interventions to reduce loneliness among dementia caregivers.

\section{Dilip V. Jeste, ${ }^{1,2,3}$ Brent Mausbach $^{1,2,4}$ AND ELLEN E. LeE ${ }^{1,2,4}$}

${ }^{1}$ Department of Psychiatry, University of California San Diego, La Jolla, CA, USA

${ }^{2}$ Sam and Rose Stein Institute for Research on Aging, University of California San Diego, La Jolla, CA, USA ${ }^{3}$ Department of Neurosciences, University of California San Diego, La Jolla, CA, USA

${ }^{4}$ Veterans Affairs San Diego Healthcare System, La Jolla, CA, USA

Email: djeste@ucsd.edu
This work was supported, in part, by NIMH K23MH119375-01 (PI: Ellen E. Lee), by the Stein Institute for Research on Aging (Director: Dilip V. Jeste), and by NIA R01 AG061941 (PI: Brent Mausbach).

\section{References}

Brodaty, H. and Donkin, M. (2009). Family caregivers of people with dementia. Dialogues in Clinical Neuroscience, 11, 217-228. https://doi.org/10.31887/DCNS.2009.11.2/ hbrodaty

Clemmensen, T., Kristensen, H. and AndersenRanberg, K. (2021). Development and Field-testing of the Dementia Carer Assessment of Support Needs Tool (DeCANT). International Psychogeriatrics, 33, 405-417.

Colins, R.N. and Kishita, N. (2020). Prevalence of depression and burden among informal caregivers of people with dementia: a meta-analysis. Ageing E Society, 40, 2355-2392.

Jeste, D.V., Lee, E.E. and Cacioppo, S. (2020). Viewpoint: battling the modern behavioral epidemic of loneliness: suggestions for research and interventions. fAMA Psychiatry, 77, 553-554. https://doi.org/10.1001/ jamapsychiatry.2020.0027

Jutkowitz, E., et al. (2021). A longitudinal evaluation of family caregivers' willingness to pay for an in-home nonpharmacologic intervention for people living with dementia: results from a randomized trial. International Psychogeriatrics, 33, 419-428.

Kaddour, L. and Kishita, N. (2020). Anxiety in informal dementia carers: a meta-analysis of prevalence. Fournal of Geriatric Psychiatry and Neurology, 33, 161-172.

Madruga, M., et al. (2021). Effects of a home-based exercise program on mental health for caregivers of relatives with dementia: a randomized controlled trial. International Psychogeriatrics, 33, 359-372.

Mausbach, B.T., et al. (2013). A comparison of psychosocial outcomes in elderly Alzheimer's caregivers and noncaregivers. American fournal of Geriatric Psychiatry, 21, 5-13.

Moore, R.C., et al. (2013). A randomized clinical trial of Behavioral Activation (BA) therapy for improving psychological and physical health in dementia caregivers: results of the Pleasant Events Program (PEP). Behaviour Research and Therapy, 51, 623-632.

Murfield, J., et al. (2021). Validity of the compassionate engagement and action scales with family carers of older adults: confirmatory factor analyses. International Psychogeriatrics, 33, 373-383.

Pione, R., et al. (2021). A psychometric appraisal of positive psychology outcome measures in use with carers of people living with dementia: a systematic review. International Psychogeriatrics, 33, 385-404.

Potier, F., Degryse, J., and de Saint-Hubert, M. (2018). Impact of caregiving for older people and pro-inflammatory biomarkers among caregivers: a systematic review. Aging Clinical and Experimental Research, 30, 119-132. 
Teahan, Á., et al. (2021). An analysis of carer burden among family carers of people with and without dementia in Ireland. International Psychogeriatrics, 33, 347-358.

Terum, T.M., et al. (2021). The association between aspects of carer distress and time until nursing home admission in persons with Alzheimer's disease and dementia with Lewy bodies. International Psychogeriatrics, 33, 337-345.
Victor, C.R., et al. (2020). The prevalence and predictors of loneliness in caregivers of people with dementia: findings from the IDEAL programme. Aging and Mental Health. https://doi.org/10.1080/13607863.2020.1753014.

von Känel, R., et al. (2020). Effects of psychosocial interventions and caregiving stress on cardiovascular biomarkers in family dementia caregivers: the UCSD Pleasant Events Program (PEP) Randomized Controlled Trial. fournals of Gerontology Series A, 75, 2215-2223. 